\title{
Un corpus phraséologique bilingue (français-italien) d'Ancien Régime : le manuscrit de Louis Goudar.
}

\author{
Lillo, Jacqueline \\ Université de Palerme \\ jacqueline.lillo@unipa.it
}

\section{Introduction}

L'auteur de la présentation à La parole exemplaire. Introduction à une étude linguistique des proverbes signale, dès la première page, la difficulté de trouver des « corpus d'époques antérieures » (2012:9). La difficulté semble être moindre si l'on recherche dans le domaine des corpus bilingues et, pour ce qui est de notre étude, dans un recueil phraséologique bilingue français/italien.

Le corpus dont il est question ici est un manuscrit qui m'a été gracieusement offert par Pierre Enckell lorsque, pour des raisons personnelles, il a dû se défaire de sa bibliothèque.

Après la description du manuscrit et la présentation de son auteur, célèbre pour sa grammaire destinée à l'enseignement du français à un public italophone, nous nous proposons de rechercher les sources de ce texte dans les dictionnaires monolingues et bilingues les plus connus de l'époque et dans des textes littéraires. L'étude de la nomenclature et des différents articles complètera la présentation.

\section{L'auteur, la Grammatica [...]}

\subsection{L'auteur}

Beaucoup d'encre a coulé sur l'identité de l'auteur, Louis Goudar, qui a souvent été confondu avec son frère Ange, «aventurier polygraphe et ami de Casanova » (Mars 1966:1). Mais leurs œuvres sont tellement différentes que, malgré quelques confusions de biographes et de certains de leurs contemporains, nous partageons les affirmations de Mars (1966:2-3) selon lesquelles il s'agit bien de deux frères, Louis étant le cadet d'un an.

Louis, ou Ludovico ou encore Lodovico, est né (1709) et mort (1782) à Montpellier. « Il réside à Milan en 1743 et publie à Venise, en 1750, une Grammatica francese à l'usage des Italiens qui aura de nombreuses rééditions » (Mars 1966:3).

\subsection{La Grammatica [...]}

Et, en effet, le succès de cette grammaire a été tout simplement fulgurant à l'époque. Mars se trompe cependant sur la date de première édition : 1744 (et non 1750) à Milan, chez Francesco Agnelli ${ }^{1}$. Les réimpressions et rééditions sont innombrables (37, de la vie de l'auteur, 276, jusqu'en 1925 !) (Lillo 1991: 121-163).

Ces grammaires reprennent les sections présentes dans les autres manuels de l'époque pour l'enseignement du français, tels ceux de Giovanni Veneroni, Michel Feri de la Salle, Jean Charles Munier, etc.; ces sections sont l'« orthographe », la «prononciation», les "parties de l'oraison» (morphologie), le «vocabulaire domestique », les «dialogues familiers", des «lettres familières » et, 
suivant le cas, des «petites histoires » et de la phraséologie (maximes et proverbes, phrases faites, etc.). Les premières éditions de la grammaire de Goudar ne possèdent pas de phraséologie qui n'apparait qu'à partir de 1766 (Minerva, Pellandra 1997: passim); de toute façon nous pouvons affirmer que la phraséologie présente dans cette dernière édition est différente de celle du manuscrit : cette grammaire, publiée à Naples, est en fait une contrefaçon réalisée par Gian Francesco Borel (Minerva, Pellandra 1997 : 62). En revanche, dans la première édition de 1744 (Milan), ou celle de 1762 (Venise), certaines expressions du dictionnaire phraséologique manuscrit objet de notre recherche se retrouvent dans les dialogues ou les regroupements thématiques (

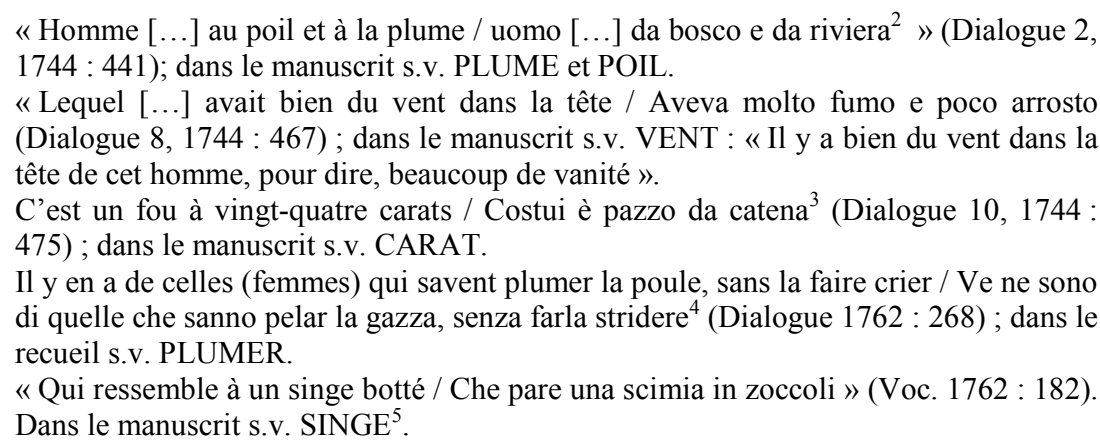

\section{Le manuscrit}

Le manuscrit a $21,5 \mathrm{~cm}$ de large, $29,5 \mathrm{~cm}$ de long et $9 \mathrm{~cm}$ d'épaisseur. Le volume in- $4^{\circ}$ est constitué de treize cahiers cousus avec une couverture très grossière de carton marron. Sur la page de titre on peut lire :

Les Richesses de La Langue Italienne, Renfermées dans un Recueil qui contient toutes les façons de parler figurées et Proverbiales de la Langue françoise, rendues en Langue Toscane.

A la fin duquel on a ajouté Cent-Cinquante Dialogues Familiers, François et Italiens sur toute sorte de sujets, à l'usage de ceux qui veulent parler le pur Toscan.

Par (le feuillet a été découpé à cet endroit) Citoyen de Montpellier

Le Ricchezze della Lingua Italiana, Racchiuse in una Raccolta laquale contiene tutt'i modi di dire figurati, e Proverbiali della Lingua francese, traslati in Lingua Toscana.

Con aggiunta in fine di Cencinquanta (sic) Dialoghi familiari, francesi, ed Italiani, sopra ogni sorta di soggetti, ad uso di chi vuol favellare il pretto Toscano.

$D a$ (le feuillet a encore été découpé à cet endroit) Cittadino Monspeliense.

Comme nous venons de le signaler, le nom de l'auteur a été volontairement éliminé du titre. Cependant, à la première page de la nomenclature, sous une languette de papier (sur laquelle est écrit Recueil de toutes les façons de Parler figurées, et proverbiales de la Langue française rendues en langue toscane), que nous avons décollée, il a été possible de lire : Proverbes françois rendus en Langue Toscane par Louis Goudar, Citoyen de Montpellier. Nous ne savons pas expliquer cette volonté de cacher le nom de l'auteur.

Les premières pages (de garde, du titre) ne sont pas numérotées. La numérotation ne commence qu'avec la première page des entrées. Seules les pages impaires sont numérotées et le mot «FIN » figure à la page 342. Sur les pages de gauche (paires), l'auteur a introduit des corrections, des exemples; il a ajouté des entrées manquantes; certaines de ces pages sont donc totalement vides. Neuf feuillets blancs clôturent le volume.

La datation est facilitée par la biographie de l'auteur (il est mort en 1782) et l'orthographe. Pour ce qui est du texte français, l'auteur modernise l'orthographe à l'exception du oi (mais l'Académie ne le fait pas dans son édition de 1762, et même pas dans son édition de 1798) et à l'exception des deuxièmes 
personnes du pluriel pour lesquelles Goudar maintient une graphie ancienne (Vous me la baillés belle, Comptés sur ma parole, etc.), alors que la majorité des textes imprimés de l'époque ont déjà adopté ez. Pour ce qui est du texte italien, Goudar reprend l'orthographe de la quatrième édition du Vocabolario degli Accademici della Crusca (1729-1738) avec ses nombreuses élisions (dee, quell'è, que' medicamenti), l'utilisation occasionnelle du $j$ à la place du $i$ (servizj), etc. et l'introduction de certains néologismes de forme ou expressions figées qui ne figurent que dans cette édition (sciabla pour sciabola, (sabre), ou encore levarsi i moscherini dal naso pour avoir du sang aux ongles). Nous pensons donc que le texte a été rédigé entre les années 1738 et 1782.

Ce dictionnaire ne présente pas de paratexte à l'exception de la page de titre signalée.

Contrairement à ce qui est anticipé dans le titre, et comme cela se produit dans toutes les grammaires prérévolutionnaires de Goudar, le manuscrit ne propose aucun dialogue à la fin du dictionnaire.

Quel est le public potentiel de ce texte ? Le titre en lui-même est assez énigmatique : « Les richesses de la langue italienne renfermées dans un recueil qui contient toutes les façons de parler figurées et proverbiales de la langue françoise, rendues en langue toscane ». À qui s'adresse-t-il, à des francophones ? à des italophones? En revanche les dialogues annoncés dans le titre, mais non inclus dans le manuscrit, sont clairement destinés à des apprenants d'italien («à l'usage de ceux qui veulent parler le pur toscan »). Il est donc très probable que tout le recueil leur soit adressé, d'autant plus que Goudar s'est assuré, grâce à sa grammaire italienne et française, une clientèle enthousiaste et fidèle essentiellement en Italie.

\section{Les sources des Richesses [...]}

Le dictionnaire bilingue (ou trilingue, si l'on prend en compte le latin) le plus répandu au XVIII ${ }^{\mathrm{e}}$ siècle est celui de l'abbé Annibale Antonini qui, pour la partie italien - (latin) - français, cite le Vocabolario degli Accademici della Crusca, et pour la partie français - (latin) - italien, celui de l'Académie française. Francesco Alberti di Villanuova fait de même en 1771 pour son célèbre Nouveau dictionnaire françoisitalien, composé sur les dictionnaires de l'Académie de France et de la Crusca (Paris : Boudet, Durand, Le Jai, Delalain ; Marseille : Mossy) qui supplante très rapidement son prédecesseur. Tous deux, Antonini et Alberti, se référent aux deux dictionnaires institutionnels qui représentent les incontournables « autorités ».

\subsection{Le Dictionnaire de l'Académie française}

Sans citer personne, on voit Goudar piller abondamment le Dictionnaire de l'Académie. Trois ou quatre éditions en avaient déjà été publiées à la date probable de rédaction de notre Recueil (1694, 1718, 1740, 1762). Les éditions de 1718,1740 et 1762 , quasiment identiques entre elles pour cette entrée, donnent pour ABOYER :

ABOYER. v. n. Japper. Il ne se dit au propre que du chien. Un chien qui aboie à la Lune. Un chien qui aboie aux voleurs. Un chien qui aboie contre tous les passans. Un chien qui aboie après tout le monde.

On dit prov. \& fig. Tous les chiens qui aboient, ne mordent pas, pour dire, Que tous ceux qui menacent ne font pas toûjours du mal.

Aboyer, au figuré, signifie Crier après quelqu'un, le presser, le poursuivre importunément. Tous ses créanciers aboient après lui.

On dit aussi fig. Aboyer après quelque chose, pour dire, La desirer, la poursuivre ardemment. Ils sont trois ou quatre qui aboient après cette charge. Aboyer après une succession.

Et on dit prov. \& fig. d'un homme qui crie inutilement contre un plus puissant que lui, que c'est aboyer à la Lune.

Aboyé, ée, part. Il n'est guéres en usage qu'au figuré. Un débiteur aboyé de tous ses créanciers (éd. 1740). 
Goudar élimine toujours les indications grammaticales, souvent les sens propre et figuré, et ne retient que la phraséologie :

\begin{abstract}
ABOYER. Tous les chiens qui aboyent ne mordent pas. Ogni cane che abbaja non $m_{0} d e^{6}$, si dice di chi fa molte parole, e pochi fatti. C'est aboyer à la Lune cela se dit à un homme qui crie inutilement contre un plus puissant que lui. Quell' è abbajare alla Luna; dicesi a chi inutilmente grida contra un più potente di se.
\end{abstract}

Il procède de la sorte dans environ $72 \%$ des articles du Recueil, (le corpus qui nous a permis de déterminer ce pourcentage, et ceux qui suivent, est constitué des vingt-cinq premières entrées des lettres $\mathrm{A}, \mathrm{H}, \mathrm{M}$ et $\mathrm{S}$. Même s'il peut sembler exigu, ce corpus est quand même représentatif de l'ensemble de l'ouvrage).

Goudar crée aussi ses propres exemples. Ainsi, alors que l'Académie (éds 1740, 1762) donne pour ABRI :

$\mathrm{ABRI}$, se dit aussi figurément de quelque lieu que ce soit où l'on est en sûreté, \& généralement de tout ce qui nous met hors de danger. La solitude est un abri contre les embarras du monde. La pauvreté volontaire est un abri contre la cupidité.

Goudar préfère :

Un homme sans abri est un oiseau sans nid. Un uomo senza ricovero è un uccello senza nido.

Ou encore pour MACHINE, alors que l'Académie propose :

MACHINE se dit aussi figurément d'une invention, d'une intrigue, d'une ruse, d'une adresse d'esprit dont on se sert dans quelque affaire. Voyez quelle machine il a fait jouer dans cette affaire. Il a remué toutes sortes de machines pour parvenir à ses fins. Quelles machines n'y a-t-il pas employées? Il a bien fallu des machines pour cela.

Goudar met :

MACHINE : Voilà la machine qu'il a fait jouer pour séduire cette pauvre fille. Ecco lo stratagemma ch'egli ha usato per sedurre quella povera giovane.

Et pour MAJESTÉ, Goudar met au féminin la phrase tirée de l'Académie (qui parlait d'un « Roi ») : «Elle a un air, un port, une majesté digne d'une Reine. Colei ha un'aria, un portamento, una maestà degna d'una Regina ».

D’une façon générale aussi, il choisit la forme discursive plutôt que la forme infinitive de l'Académie «On dit famil. Mettre quelqu'un au sac, pour dire, Le convaincre, le mettre hors d'état de pouvoir répondre » (Acad., s.v. SAC). Alors que dans notre Recueil on peut lire : «Il n'est pas profond en pareille matière, il vous sera aisé de le mettre au sac. Egli non è profondo in sì fatta materia, a lei riuscirà facile il metterlo in sacco, cioè il convincerlo cogli argomenti », s.v. SAC.

En passant du Dictionnaire de l'Académie à son propre recueil, Goudar élimine toutes les marques diastratiques, diaphasiques, diatechniques et autres (familièrement, proverbialement, bassement, figurément, en jurisprudence, etc.) qui abondent dans ce dictionnaire (Dagenais 2003, Poteaux-Dagenais 2008). Il n'en reste pas moins que le recueil maintient ce caractère de langue courante et non littéraire.

\title{
4.2 Le Vocabolario degli Accademici della Crusca
}

Le Vocabolario degli Accademici delle Crusca, à l'époque présumée de rédaction du texte qui nous intéresse, en est à sa quatrième édition? 
Goudar fait une utilisation beaucoup plus modérée de ce dictionnaire : 19\% seulement des articles reprennent, le plus souvent partiellement, des exemples ou définitions du Vocabolario, car l'objectif de Goudar est essentiellement la présentation de la phraséologie française.

La raison de ce sous-emploi est bien simple. Le Vocabolario a été construit essentiellement sur la base de l'italien littéraire, sur la langue de Dante, Boccace, Pétrarque et ensuite des plus grands auteurs des siècles successifs. Chaque entrée y est justifiée par une ou plusieurs citations référencées de la littérature italienne, grecque ou latine; cela est valable aussi pour les proverbes cités : « La lingua batte dove il dente duole ${ }^{8}$. Salvin disc. $1.295^{9} »$. Et cela est valable aussi pour Boccace. La locution italienne scuotere il pelliccione, que Goudar traduit par « en donner tout du long de l'aune », plutôt triviale, existe dans le Vocabolario degli Accademici della Crusca uniquement parce que les académiciens peuvent en citer la référence. Dès la première édition du Vocabolario, ils en donnent la définition et un exemple :

E scuotere il pelliccione, si dice dell'uso dell'atto venereo, per ricoprir la disonestà.

E Bocc. Nov. 100. $33^{10}$. S'avesse sì ad un altro fatto scuotere il pelliccion, che riuscita ne fusse una bella roba.

\subsection{Boccace}

Il n'est pas rare au $\mathrm{XVI}^{\mathrm{e}}$ et $\mathrm{XVII}^{\mathrm{e}}$ siècles de voir des auteurs de dictionnaires, même bilingues : Pierre Canal $^{11}$, Nathanaël Duez (1660, Leyde : Elsevier), Polaccho-Veneroni (Venise, Bettinelli, 1756 ; etc.), prendre comme modèle (ou déclarer prendre comme modèle) la langue des «Trois Couronnes » du XIV siècle : Dante, Boccace et Pétrarque auxquels on ajoute le plus souvent L'Arioste.

Goudar tire de nombreuses phrases du Décaméron (sept dans le corpus décrit précédemment). Apparemment, les traductions françaises disponibles sur le marché avant 1782 sont toutes d'Antoine Le Maçon ; la première édition de ce traducteur date de 1545 :

Frere Albert cogneut incontinent que ceste dame auait vng peu le cerueau creux : et faisant son compte que cestait vng vray gibier pour son oyseau, il en devint soudainement amoureux oultre mesure (Quatrième journée, deuxième nouvelle).

Les suivantes $(1670,1757)$, toujours du même traducteur, sont identiques et seule l'orthographe a été modernisée.

Frere Albert connut incontinent que cette dame avait un peu le cerveau creu : \& faisant son compte que c'estait un vrai gibier pour son oiseau, il en devint soudainement amoureux outre mesure.

Mais on constate que Goudar, qui cite cette phrase dans son texte, en propose une traduction parfaitement autonome introduisant ou éliminant des métaphores ( cerveau creu » devient «imbécile » et « un vrai gibier pour son oiseau » devient « terrain [...] propre à être cultivé par ses outils »).

Frère Albert connut d'abord que c'étoit une imbecille, et le terrein lui paroissant propre à être cultivé par ses outils, il en devint tout de suite éperdument amoureux. Frate Alberto conobbe incontamente, che costei sentiva dello scemo, e parendogli terreno da' ferri suoi, di lei subitamente, e oltre modo s'innamorò (s.v. OUTIL).

Goudar répète les phrases de Boccace quand il voit la nécessité de les mettre sous deux entrées différentes :

Tandis que tu croyois d'avoir cette nuit un jeune homme qui t'en donnât tout du long de l'aune, tu n'as eu qu'un dormeur. Dove tu credesti questa notte un giovane avere, che molto bene il pelliccione ti scotesse, avesti un dormiglione. s. v. AUNE et DORMEUR.

Remarquons, notamment dans les citations de Boccace, l'utilisation d'un lexique familier et même trivial. 


\subsection{Les autres dictionnaires}

Nous avons aussi exclu des sources possibles un dictionnaire très répandu à l'époque (une trentaine d'éditions retrouvées) : celui de Giovanni Veneroni, qu'il s'agisse de la première édition (1681) ou d'éditions successives (par exemple Veneroni-Placardi, Tourneisen : Bâle, 1750) parce qu'il présente en fait peu de phraséologie. De même, Goudar n'a pas pris en considération les Recherches italiennes et française d'Antoine Oudin (1640) qui se propose pourtant de donner «outre les mots ordinaires, une quantité de proverbes et de phrases $»$.

\subsection{Citations sacrées ou littéraires}

4.5.1 Les textes sacrés sont prédominants dans la culture de l'homme du XVIII' siècle mais Goudar en fait une utilisation relativement peu diffuse : « Souviens-toi que tu es poussière » s.v. POUSSIÈRE.

Il reprend aussi un texte de Métastase qui s'inspire du livre biblique de Judith :

C'est toi seul que j'adore, Esprit infini, source de vie, de vérité, en qui se meut, de qui dépend tout ce que l'éternité comprend. Te solo adoro, Mente infinita, fonte di vita, di verità, in cui si muove, da cui dipende quanto comprende l'eternità (s.v. SOURCE).

4.5.2 La mythologie ne peut évidemment pas être absente :

Comment approcher d'une fille qui est gardée par un dragon plus vigilant que celui qui gardait le jardin des Hespérides? Come mai accostarsi a una fancciulla, la quale è custodita da un dragone più vigilante di quello che guardava l'orto dell'Esperidi? (s.v. DRAGON).

4.5.3 Comme auteurs littéraires, en plus de Boccace omniprésent, on reconnaît au passage l'alexandrin : "A vaincre sans péril, on triomphe sans gloire", cité deux fois s.v. VAINCRE et TRIOMPHE. Certaines phrases évoquent le courant précieux par le choix du lexique ou de la syntaxe ou citent des noms connus : Iphigénie (la pièce de Racine ?) (s.v. RÔLE) ou Voltaire : «Voltaire est un Poëte de renom, de grand renom. Voltaire è un Poeta di grido, di gran nome » s.v. RENOM.

\section{$5 \quad$ La macrostructure}

\subsection{La nomenclature}

La nomenclature comprend 4083 entrées en français, réparties de façon très inégale entre les différentes lettres de l'alphabet, à l'exception, bien sûr, de « $\mathrm{W}$ », « $\mathrm{X}$ » et « $\mathrm{Y}$ » puisque « $\mathrm{X}$ » et « $\mathrm{Y}$ » commencent à peine à faire timidement leur apparition à l'époque dans les dictionnaires bilingues ${ }^{12}$ et qu'il faudra attendre le siècle suivant pour y voir l'introduction du «W». Cependant, et contrairement aux dictionnaires d'Ancien Régime d'Annibale Antonini et de Francesco d'Alberti di Villanuova, par exemple, les lettres ramistes « $\mathrm{I}$ » et « $\mathrm{J} »$, « $\mathrm{U}$ » et « $\mathrm{V}$ » sont nettement séparées dans notre manuscrit (même si le «I » n'a que neuf entrées et le « $U$ » n'en a que six contre quarante-six entrées pour le « $J$ » et cent quatre-vingt une pour le « $\mathrm{V} »)$.

Les homographes sont dégroupés (FIER, MANCHE, OUVRIER, POUDRE, POSTE, QUESTION, etc.) et on arrive, par exemple, à avoir huit sémantismes différents pour le mot TRAIT :

TRAIT. Vîte comme un trait d'arbalète. Veloce come un tiro di balestra. Etc.

TRAIT. Son image me suit par tout, j'ai toujours devant les yeux les traits de son visage. La di lei effigie mi siegue in ogni luogo, sempre ho davanti agli occhi $i$ delineamenti del suo volto. Etc.

TRAIT. Ce n'est point là un trait d'ami. Quello non è un procedere da amico. Etc.

TRAIT. Il y a de beaux traits dans ce Poëme. In quel Poema, vi sono dei concetti vaghi, de' concetti nobili. 


\begin{abstract}
TRAIT. Cette affaire-ci n'a point de trait à l'autre. Questo negozio non ha attenenza con quell'altro.

TRAIT. Il fallut couper les traits pour dégager le cheval du brancard. Convenne tagliar le tirelle, per distrigare il caval dalle stanghe.

TRAIT. Il a avalé cette bouteille de vin tout d'un trait. Ha mandato giù quel fiasco di vino tutto d'un fiato.

TRAIT. Les financiers s'enrichissent quelquefois d'un trait de plume. I finanzieri talvolta si fanno ricchi con un tratto di penna.
\end{abstract}

\title{
5.2 L'ordre alphabétique
}

Pour faciliter l'ordre alphabétique des expressions, l'auteur a choisi de mettre en vedette un seul mot simple, non accompagné d'article ni d'aucune autre indication grammaticale. Lorsqu'une expression a deux substantifs représentatifs, l'auteur n'hésite pas à les lemmatiser tous les deux : «C'est un grand abatteur de bois » s.v. ABATTEUR et BOIS ; «Disputer sur la pointe d'une aiguille » s.v. POINTE et AIGUILLE, etc. Cette règle cependant n'est pas suivie de façon systématique, ainsi : « Qui perd son bien perd son sang ", est uniquement s.v. SANG alors que «bien » est aussi chargé de sémantisme que « sang ». La catégorie grammaticale la plus représentée après le substantif est le verbe. Là encore, il arrive que l'auteur répète la même expression sous deux entrées différentes : "Il lui arrive souvent de mettre la charrue devant les bœufs. Spesso gli accade di mettere il carro innanzi a' buoi » s.v. METTRE et CHARRUE . Toujours pour ce qui est de la fréquence, l'adjectif suit de loin substantif et verbe. Une dernière catégorie, très peu représentée, inclut des adverbes ou locutions adverbiales (ARRIÈRE pour « en arrière », AUTANT, TROP, TARD, TRAVERS pour « à tort et à travers », etc), des adjectifs et pronoms (AUTRE, AUTRUI, TEL, etc.), une interjection : MALEPESTE.

Les lettres ramistes sont encore hésitantes. Ainsi se succèdent: AVARE, AVARICE, AUBADE, AVERTIR, AVEUGLE, AUGURE, AUNE. Dans ce cas «U » et «V » ont la même valeur et l'ordre est déterminé par le graphème qui suit.

\subsection{Sujets de prédilection}

Un grand nombre de «phrases» privilégient tout ce qui concerne la sphère sexuelle et «l'appétit concupiscible $»^{13}$ de Boccace ( Il concupiscevole appetito $\left.»\right)$, mais Goudar ne se limite pas à cet auteur, il cite d'autres phrases célèbres dont, par exemple, celle tirée de La Clizia (A.2.1) du Segretario Fiorentino (d'ailleurs aussi mentionnée par La Crusca): "Je ne suis pas encore si vieux que je ne rompisse bien une lance avec elle. Io non sono ancora si vecchio, ch'io non rompessi già una lancia con lei », et bien d'autres encore. La femme prend souvent un rôle prédominant, qui n'est malheureusement pas toujours à son avantage.

Comme dans les recueils du même type, la mort, le boire et le manger, l'argent, les rapports sociaux, les qualités et les défauts des hommes et des femmes sont les sujets récurrents.

\subsection{Les us et coutumes du temps}

Ce texte permet à Goudar de faire un tableau réaliste, parfois cru ou ironique des mœurs de son temps : «Les grands seigneurs sont comme certaines tentures de tapisserie, qui ne sont belles que lorsqu'on les regarde de loin. I gran signori sono come certe tappezzerie, le quali non sono belle che a guardarle da lontano » (s.v. TENTURE); ou encore: «Ces deux assassins furent rompus vifs en Grève. Quei due assassini furono arrotati in piazza di Greve». 


\section{Les articles}

\subsection{Présentateurs métalinguistiques et gloses}

Le plus souvent, Goudar donne la simple traduction de la phrase citée. Mais s'il pense que tout ou partie de celle-ci peut se révéler obscure au lecteur, il en élucide le sens par le biais de présentateurs métalinguistiques qui introduisent une glose. Dans le texte français ces gloses sont: Pour donner à entendre, pour dire, cela se dit (par ironie) à, etc., dans le texte en italien : vale, si dice (ironicamente) di, cioè, etc. Parfois la glose est pour le texte français, parfois pour le texte italien, d'autres fois encore pour les deux. Dans le cas qui suit :

MANGEOIRE. Il tourne le cul à la mangeoire. Egli volta il culo alla mangiatoja, vale, ch'egli fa l'opposto di quel che dovrebbe fare per conseguire il suo intento.

Goudar donne mot à mot la traduction de l'expression française « Tourner le cul à la mangeoire »; celleci n'ayant aucun sens en italien et n'ayant pas à sa disposition une locution équivalente, il en propose une glose en italien (dont la traduction est : «Il fait le contraire de ce qu'il devrait faire pour arriver à ses fins »).

En revanche, il considère nécessaire de gloser les deux expressions dans le cas qui suit :

MAIGRE. [...] A chevaux maigres vont les mouches, pour dire, qu'on fait tomber les charges plutôt sur les petits que sur les grands. Le mosche si posano addosso a' cavalli magri, vale, che gli aggravj si pongono addosso a' piccoli, anzi che a' grandi.

Beaucoup plus rare la seule explication de la phrase française :

MÂCHER. Je ne lui ai point mâcher, pour dire, je le lui ai dit sans ménagement. Io gliene ho detto a chiare note [...].

\subsection{Les façons de parler figurées et proverbiales de la langue française}

Comme nous l'avons signalé, l'auteur se propose d'enseigner au lecteur les Richesses de La Langue Italienne (ou toscane), contenues dans ce Recueil des façons de parler figurées et Proverbiales de la Langue françoise. Partant de la phraséologie française, connue de l'élite francophone et dialectophone, Goudar veut, par le biais d'une traduction soignée, introduire les richesses de la langue italienne/toscane parlée à l'époque par une minorité, la majorité de la population de la péninsule parlant les dialectes. Il va donc, dans la mesure du possible, travailler en même temps sur les deux langues et rechercher les équivalences en maintenant le même registre: "Ce serait dommage de l'éveiller, il dort comme un sabot. Sarebbe peccato adestarlo, che ha legato l'asino a buona caviglia, vale, che dorme saporitamente », s.v. SABOT.

Les frontières de cette «phraséologie » sont extrêmement étendues dans ce Recueil. On a :

- des séquences libres («Cette barre de fer maintient la charpente. Quella stanga di ferro mantiene il legname », s.v. MAINTENIR);

- des traductions ou des explications de mots simples ou composés dont le sens peut poser problème («Il portait un surtout de velours. Egli portava una sopraveste di veluto, s.v. SURTOUT »; « Le métier d'une sage-femme est d'accoucher les femmes. Il mestier di levatrice è di assistere le donne partorienti, $e$ ricogliere il parto », s.v. SAGE-FEMME; "SAISIE, est un acte juridique par lequel on saisit les biens meubles ou immeubles d'un débiteur. Sequestro, è un Atto giuridico per lo quale vengon sequestrati $i$ beni mobili, o stabili d'un debitore ») ;

- de simples métaphores ou comparaisons («Il vint une nuée de Russes qui désolèrent tout le pays. $E$ venne un nuvolo di Russi che devastarono tutto'l paese », s.v. NUÉE) ; 
- des collocations («Ce potage aux choux a l'air d'être bon. Quella minestra di cavoli mostra pur d'esser buona, s.v. AIR »; « Il faut saisir l'occasion, [...]. Bisogna valersi dell'occasione », S.v. SAISIR) ;

- des expressions figées («C'est un homme qui a du sang aux ongles, pour dire qu'il ne souffre point d'injures. Colui si sa levare i moscherini dal naso, vale, ch'egli non comporta ingiurie), s.v. SANG;

- des adages («Que le cocuage ne t’épouvante pas; quand on le sait, c’est peu de chose, quand on l'ignore, ce n'est rien. L'esser becco cornuto non ti rechi spavento; come uno lo sa, è poca cosa, come uno l'ignora non è nulla »);

- des proverbes ( «Avec le temps et la paille les nèfles mûrissent. Col tempo, e colla paglia si maturan le nespole, s.v. MÛRIR », «Les loix sont des toiles d'araignée qui n'arrêtent que les mouches et qui sont rompues par les frelons. Le leggi sono tela di ragnatelli, le quali sol trattengon le mosche, e sono rotte dai calabroni », s.v. ARAIGNÉE; ).

Parfois aussi l'objectif semble n'être que de mettre en évidence une construction syntaxique particulière (J'ai taché mon habit, je ne sais où ni comment. Io mi son macchiato il vestito, non so dove né come, s.v. TACHER).

\section{Conclusion}

Ce texte n'est pas exempt de quelques faiblesses, dont notamment les citations réitérées et insistantes de Boccace qui, par leur anachronisme linguistique, coupent parfois le rythme «allegro » du Recueil. Goudar montre un intérêt prédominant pour tout ce qui concerne la femme et la sphère de la sexualité, mais l'image qu'il en donne est par trop stéréotypée et pas toujours justifiée par le figement de la langue.

Ce texte aurait cependant mérité d'être imprimé pour la richesse et la variété de la langue qu'il offre, en français comme en italien. Les raisons de la non publication peuvent être multiples : manque d'argent, mort de l'auteur, etc. Mais l'écueil principal, selon nous, est que ce texte manquait de public potentiel, car il est difficile d'imaginer que l'élite francophone et dialectophone de la péninsule ait eu envie d'apprendre la phraséologie italienne (toscane) par le biais du français.

\section{Références bibliographiques}

Ademollo A. (1891). Un avventuriere francese in Italia nella seconda metà del settecento. Bergame: Cattaneo, successeurs de Gaffuri et Gatti

Alberti (Di Villanuova) F. (1771). Nouveau dictionnaire François-Italien, composé sur les dictionnaires de l'Académie de France et de la Crusca, enrichi de tous les Termes propres des Sciences et des Arts (...). Paris : Boudet, Durand, Le Jai, Delalain ; Marseille : Mossy.

Anscombre J.-C., Darbord B., Oddo A. dir. (2012). La parole exemplaire. Introduction à une étude linguistique des proverbes. Paris : Armand Colin.

Antonini A. (1735). Dictionnaire italien, latin et français ; contenant non seulement un Abregé du dictionnaire de la Crusca, mais encore tout ce qu'il y a de plus remarquable dans les meilleurs Lexicographes, Etymologistes, \& Glossaires, qui ont paru en differentes Langues. Dictionnaire français, latin et italien, contenant non seulement un Abregé du dictionnaire de l'Académie mais encore tout ce qu'il y a de plus remarquable dans les meilleurs Lexicographes, Etymologistes, \& Glossaires, qui ont paru en différentes Langues. Venise : Pitteri.

Boccace J. (1545). Le Decameron de Messire Iehan Bocace Florentin, Novvellement traduict d'Italien en Francoys par Maistre Anthoine Le Macon conseiller du Roy \& tresorier de lextraordinaire de ses guerres. Imp. Etienne Rosset dict Le Faucheur Libraire.

Boccace J. (1670). Le Decameron de Maistre Iean Bocace Florentin, Traduit d'Italien en Francois par Me Antoine Le Maçon conseiller du Roy \& Thresorier de l'extraordinaire de ses guerres. Paris, Rouen : Thomas Jolly. 
Boccace J. (1757-1761). Le Decameron [...]. Londres. (traducteur Antoine Le Maçon).

Dagenais L. (2003). La prolifération de la marque d'usage FAMILIER au XVIII ${ }^{\mathrm{e}}$ siècle (Académie 1718, 1740, 1762 et 1798). Actes du XVIII Congrès international de linguistique et de philologie romanes. Tubingen: Max Niemeyer, pp.163-168.

Goudar L. (1744). Nuova Grammatica Italiana e Franzese, Esattamente corretta. Aggiuntovi in fine un Vocabolario domestico, che riuscirà grato a' Leggitori. Milan: Agnelli.

Goudar L. (1762). Nuova grammatica italiana, e francese. Riveduta, corretta, ed accresciuta di alcune Storiette, e Lettere Familiari. Venise : Recurti.

Goudar L., Borel G.-F. (1766). Nuova grammatica italiana, e francese. Riveduta, corretta da molti errori, ed accresciuta di più voci francesi che hanno varj significati, con un aggiunta di molte Lettere italiane e francesi dell'Abate Antonini e di altri moderni autori. Naples : Raimondi.

Lillo J., (1990). Les grammaires de Ludovico Goudar 1744-1925. Quaderno n³0, Facoltà di Lettere, Università di Palermo, pp. 112.

Lillo J. (1991). Les éditions des grammaires de Ludovico Goudar. Documents pour l'histoire du français langue étrangère ou seconde, $\mathrm{n}^{\mathrm{o}} 8$, pp. 121-165.

Lillo J. éd. (2008). 1583-2000: Quattro secoli di lessicografia italo-francese. Repertorio analitico di dizionari bilingui. Berne : Peter Lang.

Mars F. L. (1966), Ange Goudar, cet inconnu. Casanova Gleanings. Vol. IX, pp.1-64.

Minerva N., Pellandra C. éds (1997). Insegnare il francese in Italia. Repertorio dei manuali pubblicati dal 1625 al 1860. Bologne : CLUEB.

Oudin A. (1640). Recherches italiennes et françoises ou dictionnaire contenant outre les mots ordinaires, vne quantité de prouerbes \& de phrases, pour l'intelligence de l'vne et l'autre langue. [...]. Paris : Antoine de Sommaville.

Poteaux M.-A., Dagenais L. (2008). De la $1^{e}$ à la $2^{e}$ édition du Dictionnaire de l'Académie française: marques diastratiques et diaphasiques. Barcelone : Euralex, pp.1175-1180.

Veneroni G. ( 1681). Dictionnaire italien et français, mis en lumière par Antoine Oudin, Secretaire Interprete du Roy [...] achevé, reveu, corrigé, et augmenté de quantité de mots ; de phrases ; [...] \& generalement des recherches de toutes les expressions françoises expliquées en Italien, tirées des meilleurs auteurs. Paris : Loyson.

Vocabolario degli Accademici della Crusca. Lessicografia della Crusca in rete. http://www.lessicografia.it/ (Toutes les éditions : 1612, 1623, 1691, 1729-1738, 1863-1923).

Dictionnaire de l'Académie française. Redon. (Toutes les éditions : 1694, 1718, 1740, 1762, 1798, 1835, 1878, 193235

\section{Notes}

${ }^{1}$ Nuova grammatica italiana, e franzese, di Lodovico Goudar, Natio di Montpellier. Prima edizione Esattamente corretta. Aggiuntovi in fine Un Vocabolario domestico, che riuscirà grato a' Leggitori. In Milano, MDCCXLIV. Presso Francesco Agnelli.

2 « On dit fig. \& fam. qu'un homme est au poil et à la plume, pour dire, qu'il a du talent, du génie, pour les armes \& pour les Lettres, qu'il a tout ce qu'il faut pour se distinguer en divers genres », (Alberti $1771: 660$ ).

3 « On dit prov. \& fig. qu'un homme est un sot, est un impertinent à vingt-quatre carats, pour dire, qu'il est sot, impertinent au souverain degré », (Alberti $1771: 130)$.

${ }^{4}$ « On dit prov. \& fig. de ceux qui adroitement \& sans exciter des plaintes, trouvent moyen de tirer de l'argent des personnes qui ont à faire à eux, qu'ils plument la poule sans la faire crier, sans crier », (Alberti 1771:657). 
5 Dans la grammaire le regroupement lexical est intitulé «Delle imperfezioni naturali» (Des imperfections naturelles) qui suit le regroupement «Delle bellezze dell'uomo e della donna » qui énumère toutes les beautés de l'homme et de la femme (« qui est faite au tour », « qui porte bien son bois », etc.) (Goudar $1762: 181$ )

${ }^{6}$ Pour plus de clarté nous utiliserons les caractères romains pour les citations en français du texte de Goudar et les italiques pour la traduction italienne, mais Goudar ne fait pas de différence dans son texte.

${ }^{7}$ La première édition est de 1612, la seconde de 1623, la troisième de 1691 et la quatrième de 1729-1738. Les successives sont postérieures à notre texte.

${ }^{8}$ Goudar traduit cette expression à l'article ABONDANCE : « De l'abondance du cœur la bouche parle »

${ }^{9}$ Discorsi Accademici de l’Abbé Antommaria Salvini, Florence : Giuseppe Manni, 1696 (vol.1), 1712 (vol.2), 1733 (vol.3).

${ }^{10}$ Boccace. Novella 100,33. Edition de Florence, Giunti : 1587.

${ }^{11}$ Dictionaire françois et italien [...] \& augmenté d'une infinité de mots \& manières de parler, tirees de Bocace, Petrarque, Dante, Ariosto \& autres fameux auteurs Italien. [Genève], Jacques Chouet, 1598 (et bien d'autres éditions).

${ }^{12}$ Chez Annibale Antonini (1770) « $\mathrm{X}$ » est absent et à « $\mathrm{Y}$ » il ne donne que l'adverbe $\mathrm{Y}$, les substantifs YEBLE, YEUSE et YEUX. Pour Francesco Alberti di Villanuova (1771), on n'a pas plus de dix entrées pour «X » et à peine huit pour " $\mathrm{Y}$ ». Ces deux lexicographes introduisent «I» et « $\mathrm{J}$ » en même temps, et Antonini utilise encore les lettrines (3) qui changent en fonction de l'ordre alphabétique (JAC, ..., IAM, ...).

${ }^{13} \mathrm{Ne}$ croyés pas que la houe, la bêche, les mets grossiers, et le mal-aise ôtent entièrement l'appétit concupiscible aux travailleurs de terre. Non creder già che la zappa, la vanga e le grosse vivande, e i disagi tolgano del tutto a' lavoratori della terra il concupiscevole appetito. Il Boccaccio (s.v. MAL-AISE). 